\title{
TDP1/TOP1 Ratio as a Promising Indicator for the Response of Small Cell Lung Cancer to Topotecan \\ Cornelia Meisenberg ${ }^{1}$, Simon E Ward², Peter Schmid ${ }^{3,4}$ and Sherif F El-Khamisy 1,5,6*
}

${ }^{1}$ The Wellcome Trust DNA Repair Group, Genome Damage and Stability Centre, University of Sussex, Brighton BN1 9RQ, UK

${ }^{2}$ The Sussex Translational Drug Discovery Group, University of Sussex, Brighton, UK

${ }^{3}$ Brighton and Sussex Medical School, University of Sussex, Brighton, UK (former affiliation)

${ }^{4}$ Centre of Experimental Cancer Medicine, Barts Cancer Institute, Queen Mary University London, UK

${ }^{5}$ Krebs Institute, Department of Molecular Biology and Biotechnology, University of Sheffield, Sheffield S10 2TN, UK

${ }^{6}$ Center of Genomics, Helmy Institute for Medical Sciences, Zewail City of Science and Technology, Giza, Egypt

\begin{abstract}
Background and objective: Small cell lung cancer (SCLC) is one of the most challenging tumors to treat due to high proliferation rate, early metastatic dissemination and rapid development of chemotherapy resistance. The current treatment protocols involve the use of topoisomerase 1 (TOP1) poisons such as irinotecan and topotecan in combination with platinum-based compounds. TOP1 poisons kill cancer cells by trapping TOP1 on DNA, generating lethal DNA double-strand breaks. A potential mechanism employed by cancer cells to resist killing by TOP1 poisons is to overexpress enzymes involved in the repair of TOP1-DNA breaks. Tyrosyl DNA phosphodiesterase 1 (TDP1) is a key player in this process and despite its importance, no data is currently available to correlate TDP1 protein and mRNA levels with catalytic activity in SCLC. In addition, it is not known if TDP1 and TOP1 protein levels correlate with the cellular response of SCLC to TOP1 based therapies.

Methods and results: We report a remarkable variation in TDP1 and TOP1 protein levels in a panel of SCLC cell lines. TDP1 protein level correlates well with TDP1 mRNA and TDP1 catalytic activity, as measured by two newly developed independent activity assays, suggesting the potential utility of immunohistochemistry in assessing TDP1 levels in SCLC tissues. We further demonstrate that whilst TDP1 protein level alone does not correlate with topotecan sensitivity, TDP1/TOP1 ratio correlates well with sensitivity in 8 out of 10 cell lines examined.

Conclusion: This study provides the first cellular analyses of TDP1 and TOP1 in SCLC and suggests the potential utility of TDP1/TOP1 ratio to assess the response of SCLC to topotecan. The establishment and validation of an easy-to-use TDP1 enzymatic assay in cell extracts could be exploited as a diagnostic tool in the clinic. These findings may help in stratifying patients that are likely to benefit from TOP1 poisons and TDP1 inhibitors currently under development.
\end{abstract}

Keywords: Topotecan; Small cell lung cancer; TOP1-DNA; TDP1 protein level; Enzymatic assay

\section{Introduction}

Small cell lung cancer (SCLC) accounts for approximately 10$20 \%$ of all lung cancers and is frequently associated with rapid tumor proliferation and a median survival of less than 3 months in untreated patients [1,2]. At diagnosis, the majority of patients present with extensive-stage disease (ED). SCLC is highly sensitive to chemotherapy, and a combination therapy with cisplatin/carboplatin and etoposide or the camptothecin drug, irinotecan is currently considered as the standard first-line treatment $[3,4]$. In patients with ED SCLC, chemotherapy produces response rates of $50 \%$ to $70 \%$ and median survival times of 7 to 9 months. However, most tumors will relapse after an initial response and nearly all patients will rapidly die from chemotherapy-resistant disease. The second-line treatment for relapsed SCLC further involves the use of another camptothecin-based drug, topotecan, which was shown to improve survival from 14 weeks to 26 weeks [1,5-7].

Both irinotecan and topotecan are camptothecin-based drugs. Camptothecins (CPT) promote cancer cell death by locking topoisomerase I (TOP1) on a DNA single-strand break intermediate $[8,9]$. An unrepaired TOP1-mediated DNA single-strand break is converted into the potentially toxic DNA double-strand break upon collision with the replication machinery [10]. The repair of these hampered TOP1 complexes involve proteasomal degradation of TOP1 to leave a 3'-phosphotyrosyl peptide that is further processed by the 3'-phosphodiesterase activity of TDP1 during chromosomal singlestrand break repair [11,12].

The ability of camptothecins (CPT) to kill cancer cells is dependent on both TOP1 activity on DNA and the rate of repair of TOP1-DNA breaks. Inhibition of TDP1 repair activity is therefore regarded as a promising mean for improving the efficacy of camptothecin-based drugs in the clinic [12-15]. Indeed, multiple studies have shown that inactivation or loss of TDP1 sensitises cells to CPT whereas overexpression of TDP1 promotes CPT tolerance [16-20]. Such findings additionally imply that high cellular levels of TDP1 may contribute to CPT drug resistance in cancer. Multiple drug discovery

*Corresponding author: Sherif F El- Khamisy, Krebs Institute, Department of Molecular Biology and Biotechnology, University of Sheffield, Sheffield, S10 2TN, UK Tel +44 114 222791; Fax +44 1273 678121; E-mail: S.El-Khamisy@sheffield.ac.uk

Received June 19, 2014; Accepted July 22, 2014; Published July 25, 2014

Citation: Meisenberg C, Ward SE, Schmid P, El-Khamisy SF (2014) TDP1/TOP1 Ratio as a Promising Indicator for the Response of Small Cell Lung Cancer to Topotecan. J Cancer Sci Ther 6: 258-267. doi:10.4172/1948-5956.1000280

Copyright: @ 2014 Meisenberg C, et al. This is an open-access article distributed under the terms of the Creative Commons Attribution License, which permits unrestricted use, distribution, and reproduction in any medium, provided the original author and source are credited. 
projects attempting to develop TDP1 inhibitors are currently underway and though some inhibitor structures have been identified, there are no validated cellular or in vivo models for confirming inhibitor hits or clinical trials in progress [21-24].

TOP1 levels are widely accepted to predict sensitivity to camptothecin in a large number of cancer types $[25,26]$. In contrast, little is known about the correlation between TDP1 or TDP1/TOP1 ratio and sensitivity to camptothecin-based therapies. Such an understanding would be particularly informative in selecting cancer types that could benefit from TDP1 inhibition in future clinical trials. Suitable candidates include cancers such as SCLC that are currently treated with camptothecin derivatives. Therefore, in this study we aimed to characterise both TDP1 and TOP1 status in a panel of SCLC cell lines and to examine the relationship between TDP1 transcript level, protein level, activity, and sensitivity to the camptothecin derivative, topotecan.

We report a remarkable variation in both TDP1 and TOP1 levels in SCLC lines. TDP1 protein levels correlate well with both TDP1 mRNA and TDP1 catalytic activity. We further show that whilst TDP1 protein level alone does not significantly correlate with topotecan sensitivity, TDP1/TOP1 ratio correlates well in 8 out of 10 SCLC cell lines examined, suggesting the potential utility of this ratio to assess topotecan sensitivity in SCLC.

\section{Materials and Methods}

\section{Cells}

The small cell lung cancer lines HCC33, H69, N417, H2171, H209 and $\mathrm{H} 510$ and the non-small cell lung cancer lines Hop62, Hop92, HCC78, H322M and H23 were obtained from Professor Peter Schmid. The remaining SCLC lines, H82, H187, H146 and H345 were bought from ATCC (ATCC, Virginia, USA). Mammalian cells were grown in a $37^{\circ} \mathrm{C}, 5 \% \mathrm{CO}_{2}$ incubator. The small cell lung cancer lines HCC $33, \mathrm{H} 69$, N417, H2171, H209, H510, H82 and H187 were grown in Gibco RPMI media (Life Technologies, Paisley, UK) supplemented with 10\% FCS and $2 \mathrm{mM} \mathrm{L-glutamine.} \mathrm{The} \mathrm{H146} \mathrm{cell} \mathrm{line} \mathrm{was} \mathrm{grown} \mathrm{in} \mathrm{Gibco} \mathrm{RPMI}$ supplemented with $10 \%$ FCS, 2 mM L-glutamine, 10 mM HEPES, $1 \mathrm{mM}$ sodium pyruvate, $4.5 \mathrm{~g} / \mathrm{L}$ glucose and $1.5 \mathrm{~g} / \mathrm{L}$ sodium bicarbonate. The H345 cell line was grown in Gibco RPMI supplemented with 20\% FCS, 4 mM L-glutamine, $10 \mathrm{mM}$ HEPES and $1.5 \mathrm{~g} / \mathrm{L}$ sodium bicarbonate. The non-small cell lung cancer lines Hop62, Hop92, HCC78, H322M and $\mathrm{H} 23$ were maintained in Gibco RPMI (Life Technologies, Paisley, UK) supplemented with $10 \%$ FCS and $2 \mathrm{mM} \mathrm{L}$-glutamine and the HCC193 cell line was maintained in Gibco DMEM (Life Technologies, Paisley, UK) containing $10 \%$ FCS and $2 \mathrm{mM} \mathrm{L-glutamine.} \mathrm{The} \mathrm{generation} \mathrm{and}$ characterisation of the DT40 Tdp1-/- chicken cells and the DT40 Tdp1/- chicken cells stably expressing human TDP1 have been described recently $[12,16,17,20,22-31]$. The DT40 chicken cells were maintained at $39^{\circ} \mathrm{C}, 5 \% \mathrm{CO}_{2}$, in Gibco RPMI (Life Technologies, Paisley, UK) supplemented with $10 \%$ FCS, $1 \%$ chicken serum, 100 U Penicillin, 100 $\mu \mathrm{g}$ Streptomycin, $2 \mathrm{mM}$ L-glutamine and $50 \mu \mathrm{M} \beta$-mercaptoethanol.

\section{Whole cell extract preparation}

Cells were collected by centrifugation (DT40 and small cell lung cancer suspension lines) or by scraping (non-small cell lung cancer adherent lines) and washed twice in ice cold phosphate buffered saline (PBS). Cell pellets were resuspended in ice-cold lysis buffer $(20 \mathrm{mM}$ Tris- $\mathrm{HCl} \mathrm{pH}$ 7.5, $10 \mathrm{mM}$ EDTA pH 8.0, $100 \mathrm{mM} \mathrm{NaCl}, 1 \%$ Triton $\mathrm{X}-100$ ) containing the recommended final concentration of Complete
Mini EDTA-free Protease Inhibitor Cocktail (Roche Applied Science, Burgess Hill, UK). Samples were briefly vortexed and extraction carried out for $30 \mathrm{~min}$ on ice. The lysate was cleared by centrifugation at 13,000 rpm for $10 \mathrm{~min}$ and the supernatant collected as whole cell extract (WCE). Protein concentration was calculated using Bradford assay and samples stored at $-80^{\circ} \mathrm{C}$.

\section{Western blotting}

Protein separation of WCE $(40 \mu \mathrm{g})$ was carried out by $10 \%$ SDSpolyacrylamide gel electrophoresis (PAGE) at $125 \mathrm{~V}$ for $2 \mathrm{hr}$. The separated proteins were subsequently transferred onto a Hybond-C Extra Nitrocellulose membrane (Fisher Scientific UK, Loughborough, $\mathrm{UK})$ at $25 \mathrm{~V}$ for $90 \mathrm{~min}$. Membranes were blocked in 5\% PBS-milk for $1 \mathrm{hr}$ prior to immunoblotting overnight with antibodies against TDP1 (ab4166; Abcam, Cambridge, UK), TOP1 (SC-32736; Santa Cruz Biotechnologies, California, USA) and actin (A4700; SigmaAldrich, Gillingham, UK) diluted in 5\% PBST-milk to 1:2000, 1:1000 and 1:3000, respectively. HRP-labelled polyclonal rabbit anti-mouse and polyclonal goat anti-rabbit secondary antibodies obtained from Dako (Ely, UK) were used at a 1:3000 dilution in 5\% PBST-milk. Blots were developed using the chemiluminescent detection reagent, SuperSignal West Pico Chemiluminescent Substrate (Fisher Scientific UK, Loughborough, UK).

\section{TDP1 activity assay: Gel-based assay}

The in vitro 3'-tyrosyl-DNA phosphodiesterase activity of SCLC whole cell extracts (WCE) was determined using a gel-based 3'-tyrosylDNA phosphodiesterase activity assay. Reactions were performed in $10 \mu \mathrm{L}$ reaction volumes containing assay buffer $(25 \mathrm{mM}$ HEPES, $\mathrm{pH}$ 8.0, $130 \mathrm{mM} \mathrm{KCl}, 1 \mathrm{mM}$ DTT), WCE (20 ng and $25 \mathrm{ng}$ ) and $50 \mathrm{nM}$ Cy5.5 labelled substrate oligomer containing a 3'-phosphotyrosyl group, 5'-(Cy5.5)GATCTAAAAGACT(pY)-3' (Midland Certified Reagent Company, Texas, USA). Reactions were carried out at $37^{\circ} \mathrm{C}$ for $1 \mathrm{hr}$ and stopped by addition of $10 \mu \mathrm{L}$ loading buffer $(44 \%$ deionized formamide, $2.25 \mathrm{mM}$ Tris-borate, $0.05 \mathrm{mM}$ EDTA, $0.01 \%$ xylene cyanol, $1 \%$ bromophenol blue). Samples were then heated at $90^{\circ} \mathrm{C}$ for 10 min prior to separation on a $20 \%$ Urea SequaGel (Fisher Scientific, Loughborough, UK) by gel electrophoresis at $190 \mathrm{~V}$ for $2 \mathrm{hr}$. Reaction products were visualised by gel imaging using the Fuji Film Fluor Imager FLA-5100 at $635 \mathrm{~nm}$ and bands quantified using Image J.

\section{TDP1 activity assay: Fluorescence assay}

In vitro 3'-tyrosyl-DNA phosphodiesterase activity of whole cell extracts (WCE) was determined using our recently developed fluorescence assay [21]. Briefly, assays were performed in a $15 \mu \mathrm{l}$ reaction volume containing assay buffer $(50 \mathrm{mM}$ Tris $\mathrm{pH} 8.0,5 \mathrm{mM}$ $\mathrm{MgCl}_{2}, 80 \mathrm{mM} \mathrm{KCl}, 0.05 \%$ Tween 20, 1 mM DTT), $10 \mathrm{nM}$ 13-mer substrate with a 3'-phosphotyrosyl oligonucleotide conjugated to a FITC molecule (Midland Certified Reagent Company, Texas, USA) and $2 \mu \mathrm{l}$ of the indicated amounts of WCE. Reactions were carried out at room temperature for $10 \mathrm{~min}$ prior to quenching with a mix of $30 \mu \mathrm{l}$ enhancer and $2 \mu \mathrm{l}$ sensor (Gyrasol Technologies, Kansas, USA). Fluorescence intensity was measured using a BMG Labtech Pherastar plate reader at excitation and emission wavelengths of $\lambda_{\text {ex }} 490 \mathrm{~nm}$ and $\lambda_{\mathrm{em}} 520 \mathrm{~nm}$. Recombinant human TDP1 (6.5 pM) [21] diluted in assay buffer was used as a 3'-tyrosyl-DNA phosphodiesterase activity control for the reactions between independent experiments.

\section{Quantitative PCR}

Total mRNA was purified from cells using the RNeasy kit (Qiagen, 
Crawley, UK) according to the manufacturer's instructions. The mRNA concentration was determined using the NanoDrop 1000 spectrophotometer (Nanodrop, Willmington, USA) at OD $230 \mathrm{~nm}$. cDNA was generated from $1 \mu \mathrm{g}$ mRNA using the Superscript First Strand RT-PCR system (Invitrogen, Paisley, UK) and real time PCR analysis was carried out on the MX3005p qPCR system (Agilent Technologies, Santa Clara, USA) using Blue qPCR Sybr low ROX mix (Fisher Scientific, Loughborough, UK) for cDNA amplification. The average cycle threshold for three readings per each of three repeat experiments was used in further calculations to determine the relative quantities of mRNA (RQ) according to the equation $R Q=2^{\Delta \Delta C T}$.

\section{CellTiter-Blue cell viability assay}

The CellTiter-Blue cell viability assay (Promega, Southampton, UK) was used to determine SCLC cell sensitivity to topotecan hydrochloride hydrate (Sigma-Aldrich, Gillingham, UK). Briefly, cells were seeded in triplicate at 10,000 cells per well of a 96-well plate in media containing topotecan $(0 \mathrm{nM}$ to $500 \mathrm{nM})$. The contents were mixed thoroughly and the plates incubated at $37^{\circ} \mathrm{C}$ for 4 days. At day 4 , CellTiter-Blue reagent $(20 \mu \mathrm{l})$ was added to each well, the plates incubated at $37^{\circ} \mathrm{C}$ for $2 \mathrm{hr}$ and fluorescence intensity determined using the GloMax Multi Detection System (Promega, Southamption, UK) at excitation and emission wavelengths of $\lambda_{\text {ex }} 560 \mathrm{~nm}$ and $\lambda_{\mathrm{em}} 590 \mathrm{~nm}$. Percentage viability (\%) was calculated using background-subtracted readings as:

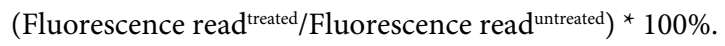

\section{Results}

TDP1 is viewed as a promising target for inhibition in cancers such as SCLC that are currently treated with camptothecin-based therapies. Since topoisomerase I (TOP1) is generally thought to dictate the sensitivity of cancers to camptothecin, we sought to determine the cellular level of both TDP1 and TOP1 in a panel of SCLC cell lines (HCC33, H69, N417, H2171, H209 and H510). Separation of $40 \mu \mathrm{g}$ of whole cell extracts (WCE) for each of the SCLC cell lines by $10 \%$ SDSPAGE and analysis by Western blotting using TDP1 and TOP 1 specific antibodies revealed a significant variation in both TDP1 and TOP1 protein level (Figure 1A-C). Interestingly, we noticed that SCLC lines possess higher levels of TDP1 compared to non-small cell lung cancer lines (HCC193, Hop62, Hop92, HCC78, H322M and H23; Figure 1A), suggesting that TDP1 may play a role in the biology, progression and, in particular, the therapeutic response of SCLC to camptothecin-based treatments.

Cellular protein levels are maintained by both transcriptional and post-translational regulation. To determine the nature of the variation in cellular TDP1 and TOP1 protein levels in SCLC, cellular mRNA levels for both TDP1 and TOP1 were determined by quantitative real-time PCR using primers designed against exon-crossing regions for TDP1 and TOP1 cDNA (Figure 2A and 2C). Since there is no real isogenic control gene between the cell lines, the relative quantifications were obtained by normalising mRNA levels to that obtained for HCC33 for TDP1 mRNA measurements, or H510 for TOP1 mRNA. We further determined the Pearson's correlation coefficient between mRNA and protein levels for both TDP1 and TOP1 to examine the relationship between mRNA and protein levels (Figure 2B and 2D). We observed a positive correlation between TDP1 mRNA and TDP1 protein ( $\mathrm{r}=0.681$, $\mathrm{p}=0.136)$ and a slightly weaker correlation between TOP1 mRNA and TOP1 protein $(\mathrm{r}=0.519, \mathrm{p}=0.292)$.

Post-translational modifications may further dictate the cellular activity of TDP1 $[11,16,18,19,32]$. It is therefore important to examine whether cellular TDP1 protein levels correlate with its activity, which is critical for designing a suitable diagnostic tool for selecting tumors that could benefit from TDP1 inhibitor-based therapies. To test this, we employed two orthogonal enzymatic assays; a gel-based assay and a fluorescence-based assay that was recently developed in our labs [21]. For the gel-based assay we engineered a 5'-Cy5.5 labelled substrate containing a 3'-phosphotyrosine modification (Figure $3 \mathrm{~A}$ ), thus introducing a unique imaging opportunity that does not involve the bothersome radioactive based methods that we used previously $[11,17,18]$. Whole cell extracts prepared from the SCLC cell lines were used as a source for TDP1 activity and reaction products were subsequently separated on a $20 \%$ denaturing PAGE by gel electrophoresis, and then visualised using a FujiFilm Fluor Imager FLA-5100 at $635 \mathrm{~nm}$ (Figure 3B). The relative activity was calculated by normalising the percentage cleavage to that obtained for $20 \mathrm{ng}$ HCC33 whole cell extract and results obtained from three biological replicates are shown in Figure 3C. The results demonstrate a positive

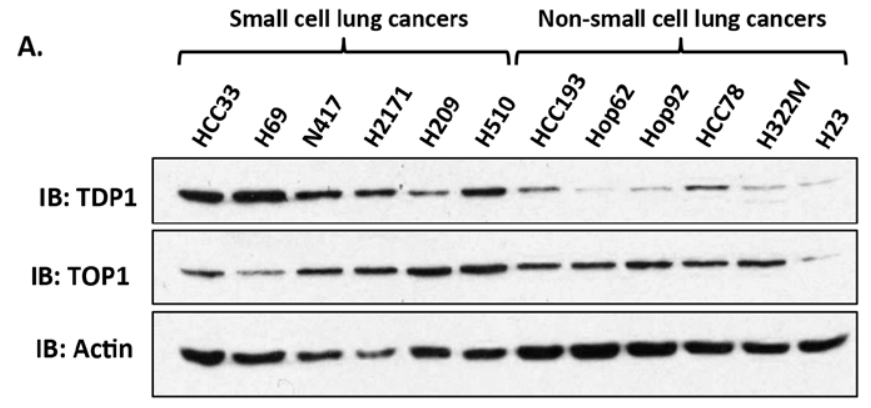

B.

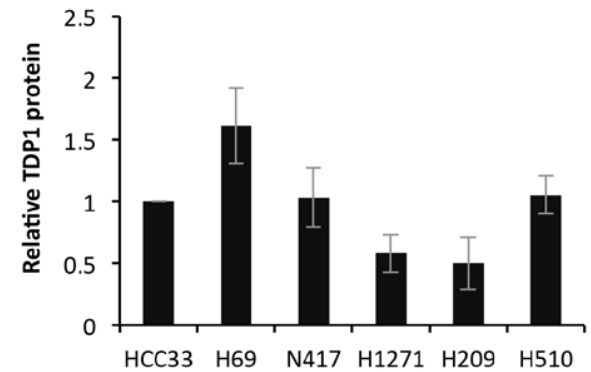

C.

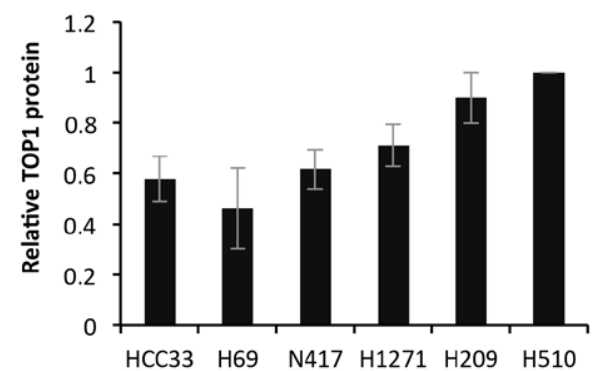

Figure 1: Small cell lung cancer cell lines display remarkable variation in both TDP1 and TOP1 protein level. (A) Whole cell extracts $(40 \mu \mathrm{g})$ from the indicated small cell lung cancer cell lines and other non-small cell lung cancer cells were separated by $10 \%$ SDS-PAGE and immunoblotted using antibodies against TDP1, TOP1 and actin. A quantitation of relative TDP1 (B) and TOP1 (C) protein level is depicted. Data are the average of 3 independent experiments \pm STD normalised to HCC33 for TDP1 and H510 for TOP1. 
A.

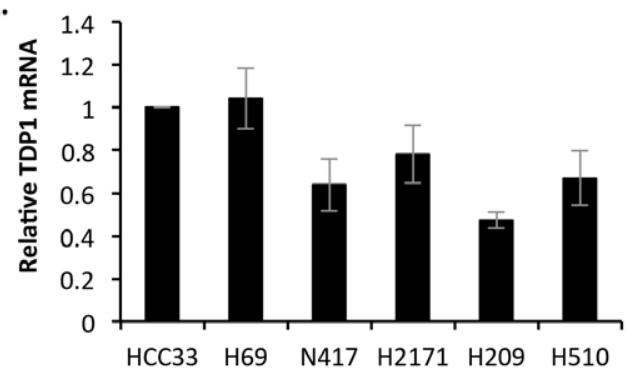

C.

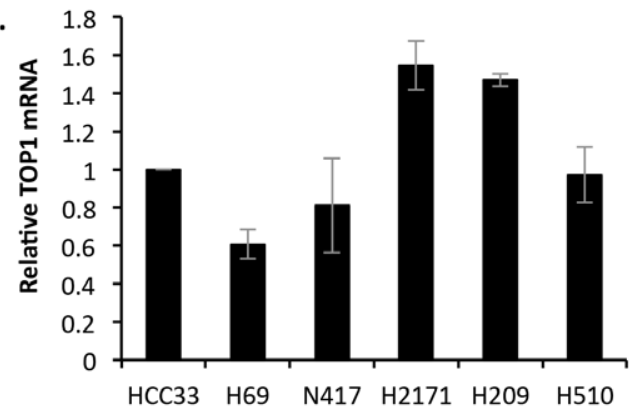

B.

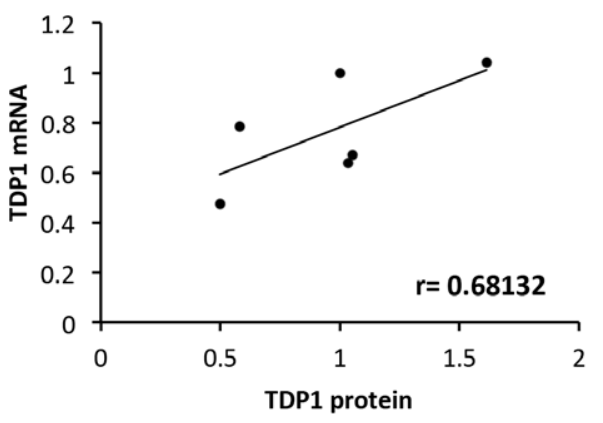

D.

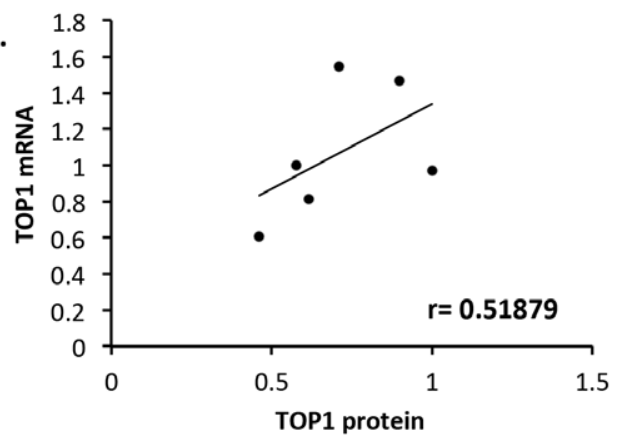

Figure 2: Small cell lung cancer cell lines display good correlation between TDP1 or TOP1 protein level and mRNA. cDNA from each of the SCLC lines was generated from $1 \mu \mathrm{g}$ purified mRNA using the Superscript First Strand RT-PCR and real time PCR analysis was conducted using TDP1 primers (A) or TOP1 primers (C) and an MX3005p qPCR system. The relative quantity of $m R N A\left(R Q\right.$ value) for each repeat experiment was calculated according to the equation $R Q=2^{\triangle \triangle C T}$ and the $R Q$ values normalised to that obtained for the HCC33 cell line. The average of normalised $R Q$ values from 3 independent experiments \pm STD is depicted (A\&C). A graphical representation of the Pearson's correlation coefficient ( $r$ ) between SCLC TDP1 protein levels and TDP1 mRNA is shown in (B) and that for SCLC TOP1 protein level and TOP1 mRNA is shown in (D).

correlation between TDP1 protein level and TDP1 activity in SCLC cell lines $(r=0.700, p=0.121$ for $20 \mathrm{ng}$ WCE; and $\mathrm{r}=0.747, \mathrm{p}=0.088$ for $25 \mathrm{ng}$ WCE) (Figure 3D and 3E). These observations are in agreement with a recent study by the Pommier group on NSCLC reporting a remarkably similar positive correlation $(\mathrm{r}=0.75)$ between TDP1 protein level and catalytic activity [33].

To better understand the relationship between TDP1 protein level and cellular activity, we additionally employed our recently developed fluorescence assay [21]. This 384-well plate format assay uses a FITC-conjugated 13-mer 3'-phosphotyrosyl substrate that releases a fluorescence signal upon processing (Figure $4 \mathrm{~A}$ ). We first examined whether this new assay would be reliable in determining TDP1 enzymatic activity in whole cell extracts. To test this, we generated chicken DT40 Tdp1-/- cells stably expressing low and high levels of human TDP1 and examined whether TDP1 level correlates with enzymatic activity. Whilst extracts from DT40 Tdp1-/- cells had no detectable TDP1 activity, incubation of the TDP1 substrate with increasing concentrations of whole cell extract prepared from DT40 Tdp1-/- cells expressing human TDP1 led to a proportional increase in TDP1 activity as measured by relative fluorescence (Figure 4B and $4 \mathrm{C})$. These observations validate the assay and suggest its robustness in determining TDP1 activity in cellular extracts. We next examined whole cell extracts $(0.25 \mathrm{ng})$ generated from the SCLC cell lines using a recombinant TDP1 protein $(6.5 \mathrm{pM})$ as a control. The relative activity obtained for each cell line (Figure 4D) was calculated by normalising the fluorescence intensity against that obtained for recombinant TDP1 for three independently generated extracts. These results illustrate a clear variation in TDP1 activity in SCLC and, importantly, the Pearson's correlation coefficient $(r=0.86, p=0.028)$ suggests a significant positive correlation between cellular TDP1 activity and protein level (Figure $4 \mathrm{E})$.

TDP1 is a key repair factor responsible for resolving TOP1-linked DNA breaks generated by camptothecin and its derivatives $[17,20]$. It was therefore of interest to understand the relationship between TDP1 levels and sensitivity of SCLC to the camptothecin drug, topotecan that is currently used in the clinic to treat relapsed SCLC. Cell viability in the presence or absence of topotecan (TPT) was determined using the CellTiter-Blue viability reagent. Cells were treated with a concentration range of topotecan ( $0 \mathrm{nM}$ to $500 \mathrm{nM}$ ) and grown for four days prior to analysis from which percentage viability (Figure 5A) and the Pearson's correlation coefficient between TDP1 protein level and topotecan sensitivity (Figure 5B) were calculated. We observed a weak positive correlation between TDP1 level and viability at all topotecan concentrations analysed (Figure 5B and Table 1). Following topotecan treatment, TDP1 exclusively repairs TOP1 mediated DNA breaks, thus we reasoned that the weak correlation might be due to variations in TOP1 levels. Indeed, subsequent analyses revealed a stronger negative correlation between TOP1 levels and topotecan sensitivity (Figure 5C), demonstrating that TOP1 is an important player in determining the response of SCLC to topotecan. To account for variations in TOP1 levels that may explain the weak correlation between TDP1 and topotecan sensitivity, we normalised TDP1 to TOP1 protein levels (TDP1/TOP1) for further analysis. This normalisation revealed a modest increase 
A.

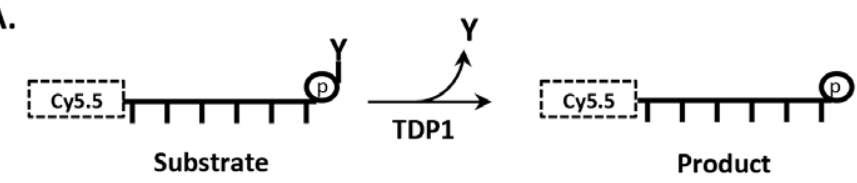

B.

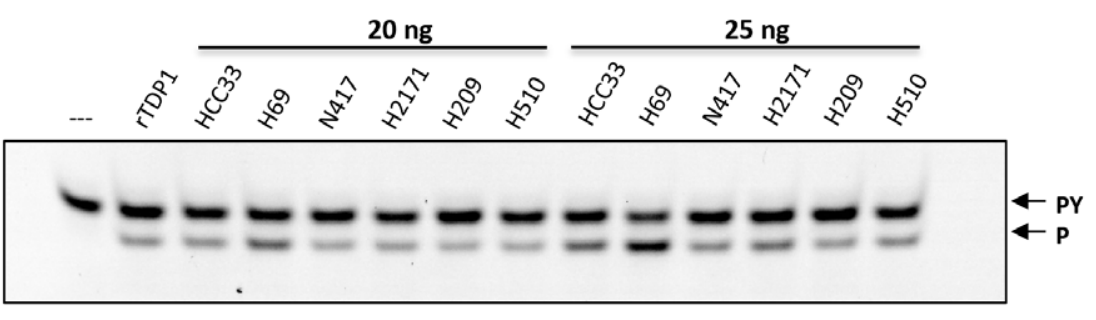

C.

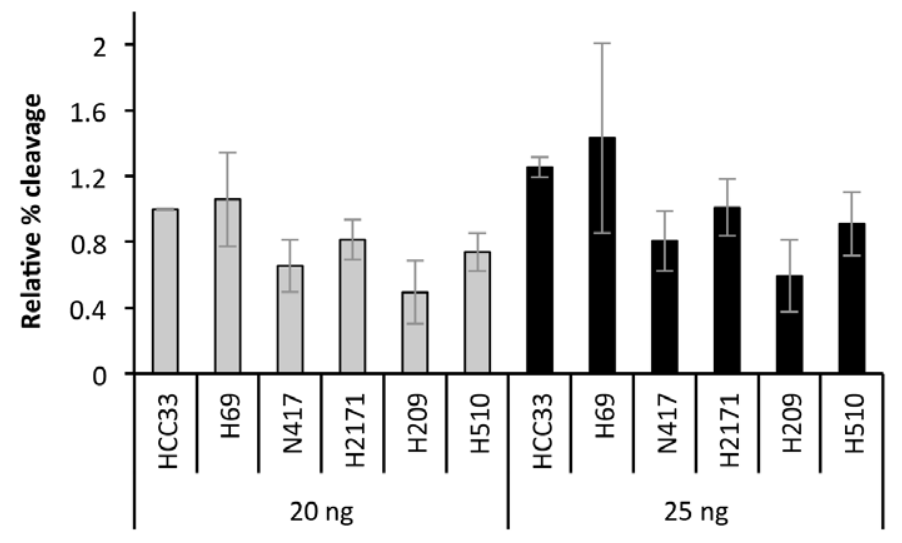

D.

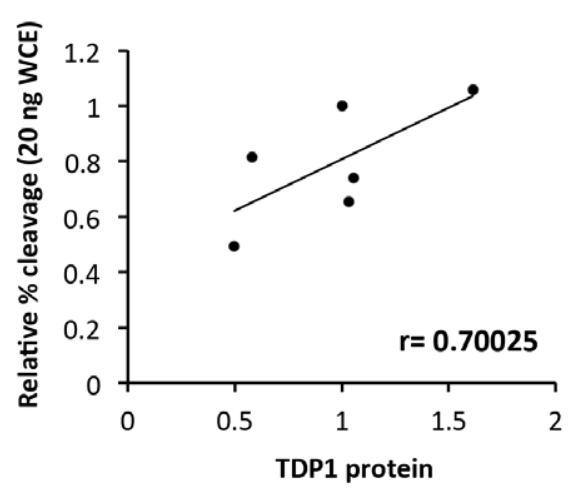

E.

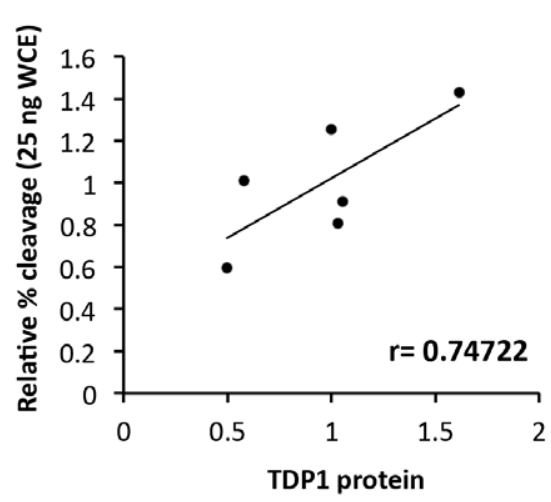

Figure 3: TDP1 catalytic activity (Gel-based assay) correlates with the level of TDP1 protein in SCLC cells. (A) Diagrammatic representation of TDP1 processing of a Cy5.5 labelled oligonucleotide harbouring a 3'-phosphotyrosine modification. The product of a TDP1 reaction is a Cy5.5 labelled oligonucleotide with 3'-phosphate. (B) The indicated amounts of WCE $(20 \mathrm{ng}$ and $25 \mathrm{ng})$ were incubated with $50 \mathrm{nM}$ substrate in assay buffer at $37^{\circ} \mathrm{C}$ for $1 \mathrm{hr}$. Reaction products were separated on a $20 \%$ Urea SequaGel by gel electrophoresis at $190 \mathrm{~V}$ for $2 \mathrm{hr}$ and visualised using a FujiFilm Fluor Imager FLA-5100 at $635 \mathrm{~nm}$. Arrows denote the position of the substrate (PY) and cleaved product $(P)$. (C) Quantification of the average relative TDP1 activity normalised to that obtained from HCC33 cell line from 3 independent experiments \pm STD. Recombinant TDP1 (8 pM) was employed as a positive control. (D) A graphical representation of the Pearson's correlation coefficient ( $r$ ) between TDP1 protein level and activity.

in the positive correlation between TDP1 and topotecan sensitivity (Figure 5D, solid line). Interestingly, the cell line H69 that expresses the highest level of TDP1 and the lowest level of TOP1 unexpectedly exhibited relatively high topotecan sensitivity. Repeating the analyses without this outlier cell line revealed a strong positive correlation between TDP1/TOP1 and topotecan sensitivity in the five remaining cell lines (Figure 5D, dotted line and Table 1). 
A.

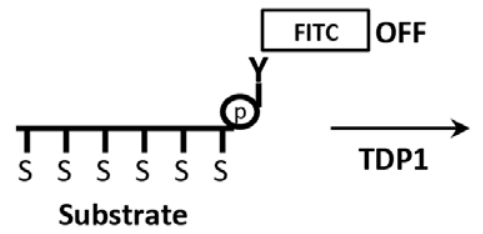

B.

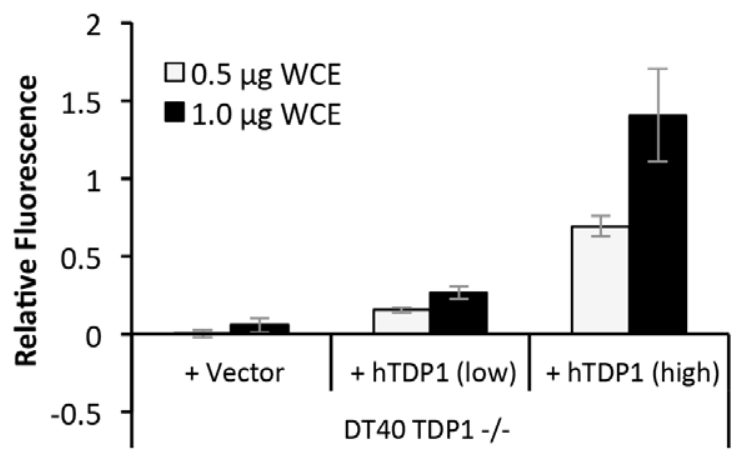

D.

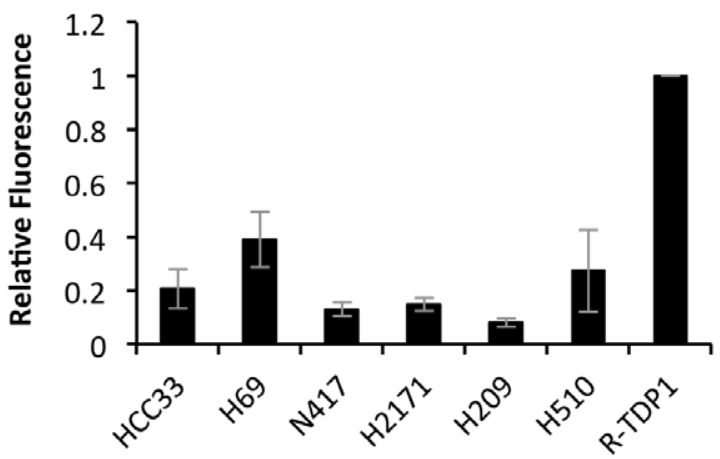

C.

DT40 TDP1 -/-

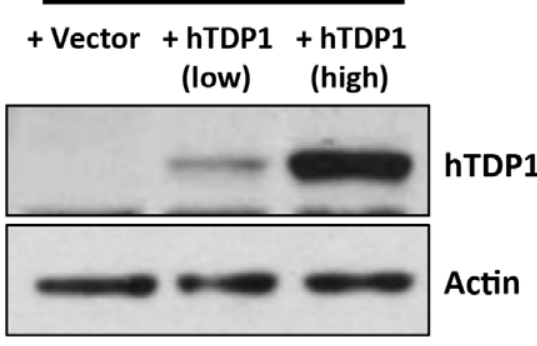

E.

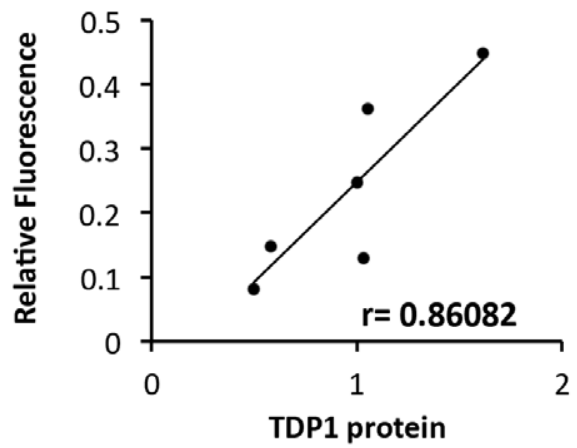

Figure 4: TDP1 catalytic activity (Fluorescence assay) correlates with the level of TDP1 protein in SCLC cells. (A) A Metal ion sensor (indicated S) along the phosphate backbone of the substrate oligomer keeps the bound FITC-conjugated tyrosine in a non-fluorescent state (OFF). During a TDP1 driven reaction, the FITC conjugated tyrosine is released and distance generated between the released FITC-tyrosine and the oligonucleotide increases, allowing for FITC fluorescence (ON) that can be detected and quantified using a fluorescence imager. (B) A $15 \mu$ final volume reaction containing $0.5 \mu \mathrm{g}$ or $1.0 \mu \mathrm{g}$ WCE from the indicated DT40 cell lines and $10 \mathrm{nM}$ substrate was incubated for $10 \mathrm{~min}$ at room temperature. Reactions were stopped by addition of a reaction quencher and fluorescence intensity was measured using a BMG Labtech Pherastar plate reader at excitation and emission wavelengths of $\lambda_{\text {ex }} 490 \mathrm{~nm}$ and $\lambda_{\text {em }} 520 \mathrm{~nm}$. Fluorescence was normalised to that obtained for recombinant human TDP1 $(6.5 \mathrm{pM})$ and data represent the average of 3 independent experiments \pm STD. (C) Whole cell extracts $(40 \mu \mathrm{g})$ from the indicated DT40 cell lines were separated by $10 \%$ SDS-PAGE and immunblotted using antibodies against TDP1 and actin. (D) A $15 \mu$ final volume reaction containing $0.5 \mu \mathrm{g}$ SCLC WCE and $10 \mathrm{nM}$ substrate was incubated for $10 \mathrm{~min}$ at room temperature. Reactions were stopped by addition of a reaction quencher and fluorescence intensity was measured using a BMG Labtech Pherastar plate reader at excitation and emission wavelengths of $\lambda_{\mathrm{ex}} 490 \mathrm{~nm}$ and $\lambda_{\mathrm{em}} 520 \mathrm{~nm}$. Average fluorescence was normalised to that obtained for recombinant human TDP1 (6.5 pM), and data represent the average of 3 independent experiments \pm STD. (E) A graphical representation of the Pearson's correlation coefficient ( $r$ ) between SCLC TDP1 protein level and TDP1 activity from $0.5 \mu \mathrm{g}$ WCE.

To examine if these findings are restricted to the 5 SCLC lines employed above, we obtained and analysed four additional SCLC lines. TDP1 and TOP1 protein level for each cell line was determined by Western blotting (Figure 6A-C) and the corresponding topotecan sensitivity was measured as percentage viability using the CellTiterBlue cell viability assay (Figure 6D). These analyses revealed that the majority of SCLC cell lines ( 8 out of 10 ) showed a strong and significant positive correlation $(\mathrm{r}=0.94139, \mathrm{p}=0.000491)$ between TDP1/TOP1 ratio and topotecan sensitivity (Figure $6 \mathrm{E}$, dotted line and Table 1) with 2 hypersensitive outliers, $\mathrm{H} 69$ and $\mathrm{H} 187$ that intriguingly possess the highest level of TDP1 (Figure 6E, solid line). These data suggest TDP1/ TOP1 ratio as a useful indicator for the response of SCLC to topotecan 


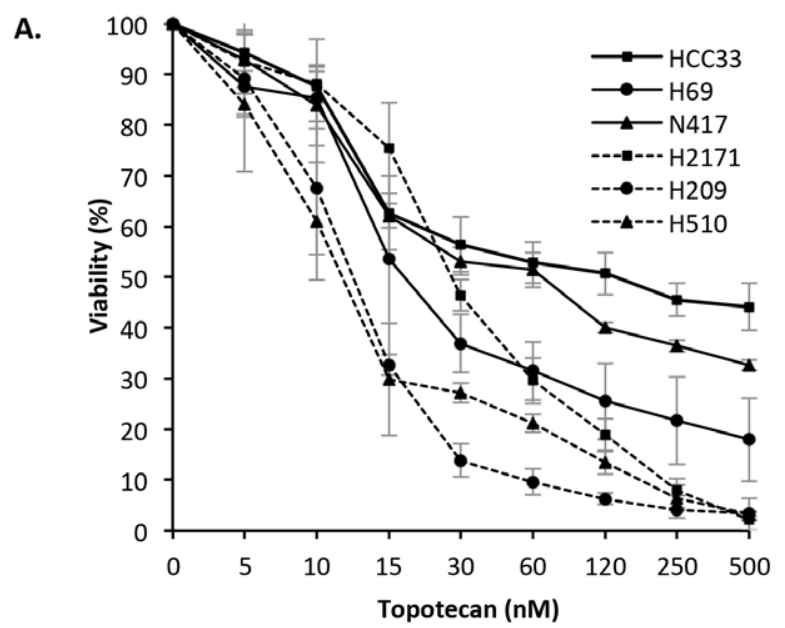

B.

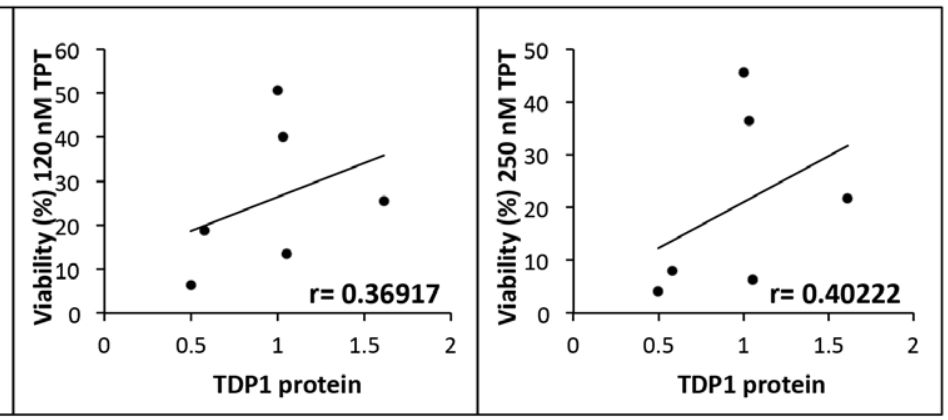

C.
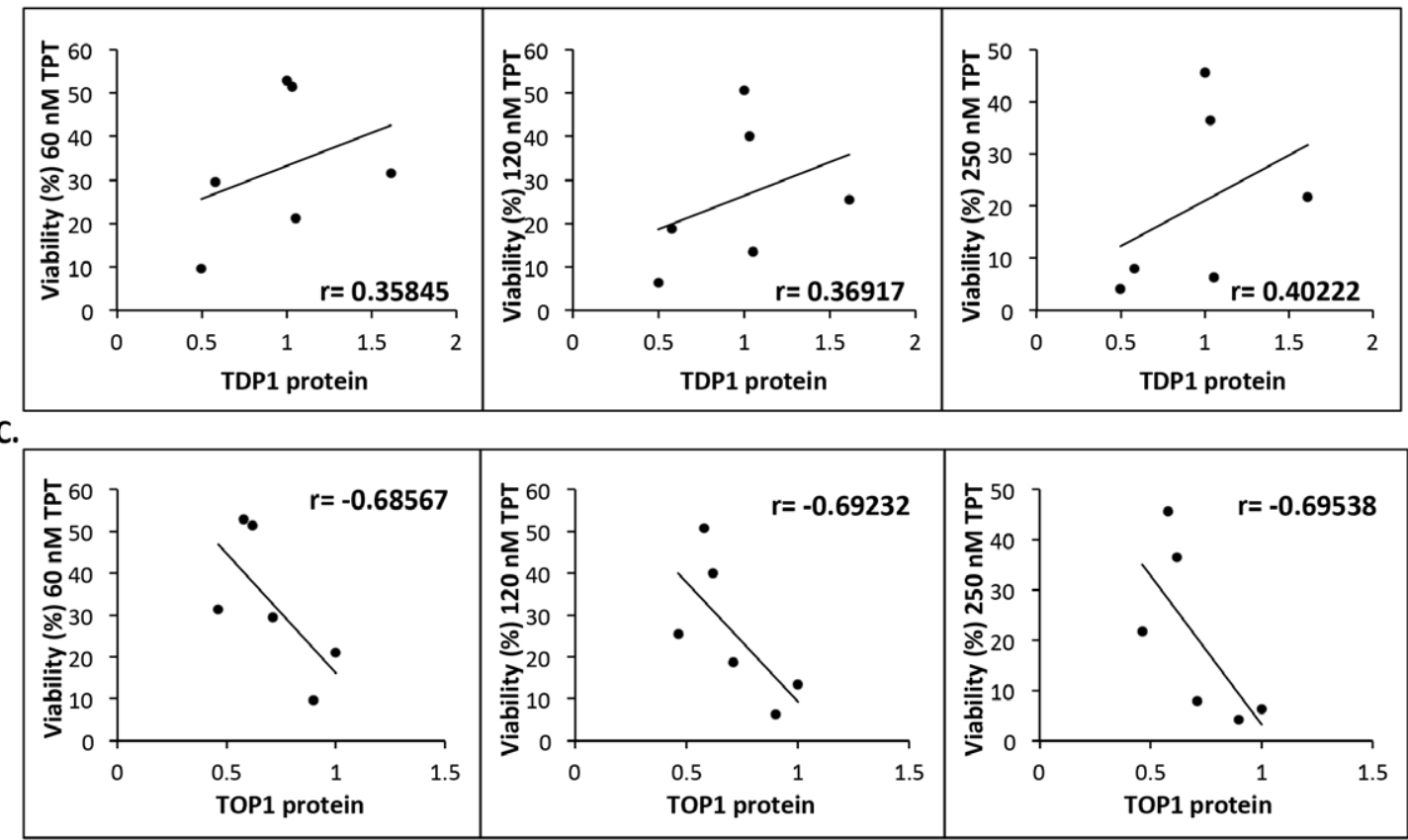

D.
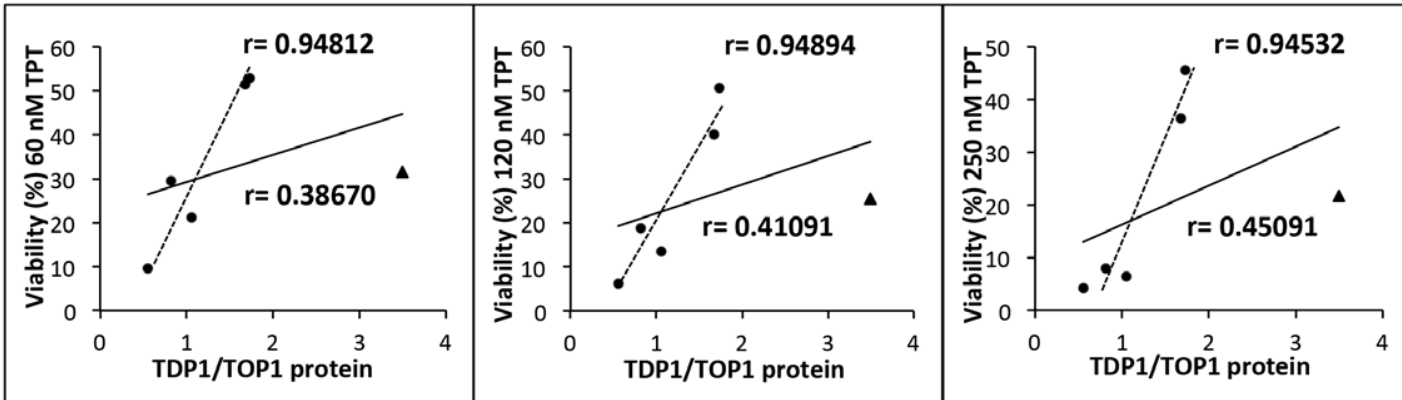

Figure 5: TDP1 and TOP1 levels modulate the cellular response of SCLC to topotecan. (A) The indicated SCLC cell lines were seeded in triplicate at 10,000 cells per well in a 96-well plate in $100 \mu \mathrm{l}$ media containing the indicated concentration of topotecan (TPT) and incubated at $37^{\circ} \mathrm{C}$ for 4 days. Viability was assessed by adding $20 \mu \mathrm{L}$ of the CellTiter-Blue viability assay reagent to wells on day 4 , followed by 2 hour incubation at $37^{\circ} \mathrm{C}$. Fluorescence was determined using a GloMax Multi Detection System (Promega, Southamption, UK) at excitation and emission wavelengths of $\lambda 560 \mathrm{~nm}$ and $\lambda .590 \mathrm{~nm}$, respectively. For each cell line, percentage viability (\%) was calculated as [(Fluorescence read $\left.{ }^{\text {treatment/Fluorescence readuntrated }}\right)^{\star} 100 \%$ ] and the graphical representation shows the results obtained from three independent experiments \pm STD. Graphical representations depicting Pearson's correlation coefficient $(r)$ between viability at the indicated TPT concentrations and TDP1 (B), TOP1 (C), or TDP1/TOP1 levels (D). The solid line represents the correlation coefficient for all six SCLC cell lines whereas the dotted line represents the correlation coefficient for the five SCLC cell lines HCC33, N417, H2171, H209 and H510, excluding H69 (triangle), which exhibits high levels of TDP1 and an unexpected hypersensitivity to topotecan. 


\begin{tabular}{|c|c|c|}
\hline & $\square$ & Probability (p) (2-tailed) \\
\hline TDP1/TOP1 and Viability (\%) 120 nM TPT, n=8 (No H69 or H187) & 0.941 & 0.000491 \\
\hline TDP1/TOP1 and Viability (\%) 120 nM TPT, n= 5 (No H69) & 0.949 & 0.014 \\
\hline TDP1/TOP1 and Viability (\%) 60 nM TPT, n=5 (No H69) & 0.948 & 0.014 \\
\hline TDP1/TOP1 and Viability (\%) 250 nM TPT, n=5 (No H69) & 0.945 & 0.154 \\
\hline TDP1 protein and TDP1 ac, vity-Gyrasol, $n=6$ & 0.861 & 0.028 \\
\hline TDP1 protein and TDP1 ac,vity-GB assay $25 \mathrm{ng}, \mathrm{n}=6$ & 0.747 & 0.088 \\
\hline TDP1 protein and TDP1 ac,vity-GB assay $20 \mathrm{ng}, \mathrm{n}=6$ & 0.703 & 0.121 \\
\hline TOP1 protein and Viability (\%) $250 \mathrm{nM}$ TPT, $\mathrm{n}=6$ & -0.695 & 0.125 \\
\hline TOP1 protein and Viability (\%) $120 \mathrm{nM}$ TPT, $\mathrm{n}=6$ & -0.692 & 0.127 \\
\hline TOP1 protein and Viability (\%) $60 \mathrm{nM}$ TPT, $\mathrm{n}=6$ & -0.686 & 0.133 \\
\hline TDP1 protein and TDP1 mRNA, $n=6$ & 0.681 & 0.136 \\
\hline TOP1 protein and Top1 mRNA, $n=6$ & 0.519 & 0.292 \\
\hline TDP1/TOP1 and Viability (\%) 250 nM TPT, n=6 & 0.451 & 0.369 \\
\hline TDP1/TOP1 and Viability (\%) 120 nM TPT, n=6 & 0.411 & 0.418 \\
\hline TDP1/TOP1 and Viability (\%) 60 nM TPT, n=6 & 0.387 & 0.448 \\
\hline TDP1 protein and Viability (\%) $250 \mathrm{nM}$ TPT, n=6 & 0.402 & 0.429 \\
\hline TDP1 protein and Viability (\%) $120 \mathrm{nM}$ TPT, n=6 & 0.369 & 0.471 \\
\hline TDP1 protein and Viability (\%) $60 \mathrm{nM}$ TPT, $\mathrm{n}=6$ & 0.358 & 0.485 \\
\hline TDP1/TOP1 and Viability (\%) 120 nM TPT, n=10 & 0.199 & 0.582 \\
\hline
\end{tabular}

Table 1: Summary of the Pearson's correlation coefficient ( $r$ ) and respective probability for all correlation analyses performed in the study.

and identify $\mathrm{H} 69$ and $\mathrm{H} 187$ cell lines as potential resources to further study the molecular basis of the repair of TOP1 breaks in SCLC.

\section{Discussion}

The highly proliferative and metastatic small cell lung cancers are routinely treated in the clinic with the camptothecin derivatives irinotecan and topotecan [7]. Though most patients initially respond to chemotherapy, virtually all patients experience rapid relapse and bad prognosis. Camptothecins induce damage to cancer cells by locking topoisomerase I (TOP1) on to DNA at single-strand breaks (SSBs) $[9,12,17,34-36]$. These SSBs can be converted to toxic DNA double-strand breaks by collision with the DNA replication machinery. Tyrosyl DNA phosphodiesterase 1 (TDP1) is a key player in the repair of TOP1 associated DNA breaks [16,18,19,37]. Loss of TDP1 has been shown to prolong the persistence of TOP1-SSBs and increases the likelihood of DNA double-strand break formation, thereby potentiating camptothecin-induced cytotoxicity. Many research groups are therefore keen to uncover small molecule TDP1 inhibitors to improve the clinical response to camptothecin-based therapies. Despite the common use of camptothecin derivatives to treat SCLC in the clinic, the impact of TOP1 and TDP1 on the response of SCLC to camptothecins remains largely unknown. Furthermore, an understanding of the relationship between cellular TDP1 protein level, transcript level and catalytic activity in SCLC is also lacking. This knowledge is important for the design of practical diagnostic tools for patient tumor characterisation.

In this study, we report a higher level of TDP1 expression in SCLC compared to other lung cancer cell lines and a remarkable variation in both TDP1 and TOP1 protein levels in SCLC. These data highlight SCLC as a key cancer type that may benefit from TDP1 inhibition. TDP1 protein level correlates well with both TDP1 mRNA and cellular TDP1 activity, as determined by two independent TDP1 activity assay methods. Although we found a good negative correlation between TOP1 levels and SCLC sensitivity to the camptothecin drug topotecan, we noted that TDP1 protein level alone did not correlate well with the sensitivity of SCLC cell lines to topotecan. Normalisation of TDP1 levels to that of TOP1 revealed a strong positive correlation to topotecan in
$80 \%$ of the SCLC cell lines employed in the study. Interestingly, the two outlier cell lines (H69 and H187) that display relatively high sensitivity to topotecan possess remarkably higher levels of TDP1 than the rest. It is worth noting that TDP1 is not the only factor involved in the cellular response to topotecan and that redundant and partially overlapping pathways driven by TDP2, MUS81, XPF-ERCC1, and MRE11 are also implicated in this process $[27,38,39]$. It is possible that the H69 and H187 cell lines lack one or more TDP1 alternative pathway for dealing with TOP1-breaks, which might account for their unexpected high sensitivity to topotecan. TDP1 posttranslational modification by phosphorylation, SUMOylation, and PARylation is important for recruitment and enrichment of TDP1 at sites of DNA damage $[11,18,19,32]$, and it is thus possible that a lack of one or more of these modifications renders TDP1 less active in vivo in $\mathrm{H} 69$ and $\mathrm{H} 187$ cells, despite its higher expression level compared to the rest of the panel. A complete understanding of why these two cell lines are hypersensitive to CPT may furthermore reveal novel synthetic lethal targets for drug design and combination therapy with camptothecin treatments and will be the focus of further studies.

The data presented here suggest the potential for utilising TDP1/ TOP1 ratio as an indicator for the response of SCLC to topotecan, however it will be important to additionally determine if this will hold true in patient SCLC tissues in future studies. Another important outcome of this study is the development and validation of an easy-touse TDP1 enzymatic assay in cell extracts, which could be exploited as a diagnostic tool in the clinic to stratify patients undergoing TOP1 based therapies. Furthermore, the strong correlation between TDP1 protein level, enzymatic activity, and mRNA level, suggest the potential utility of immunohistochemistry and microarray analysis in assessing TDP1 levels in SCLC tissues.

In summary, we report a remarkable variation in TDP1 and TOP1 levels in a panel of SCLC cell lines and suggest that SCLC could be an ideal model for confirming TDP1 inhibitor hits emerging from existing drug discovery projects and in future clinical trials. We additionally highlight the potential for TDP1/TOP1 ratio as a promising indicator for the response of SCLC to topotecan, though further work is required to confirm these findings in primary SCLC tissues. 
A.

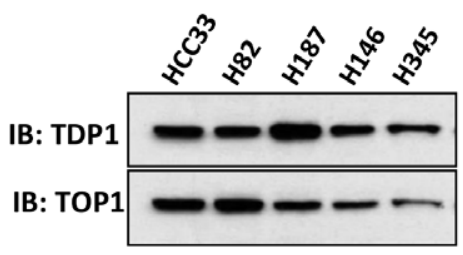

B.

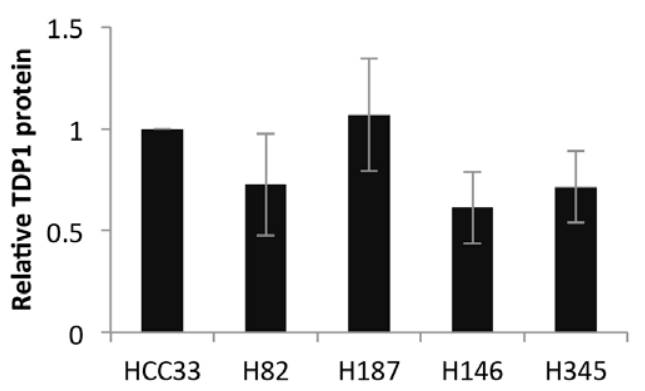

D.

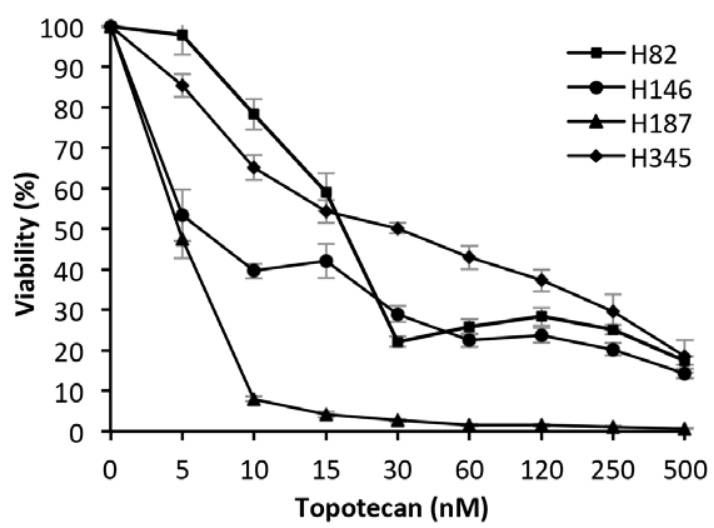

C.

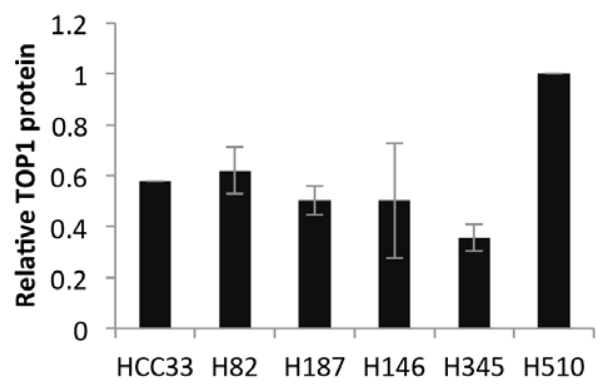

E.

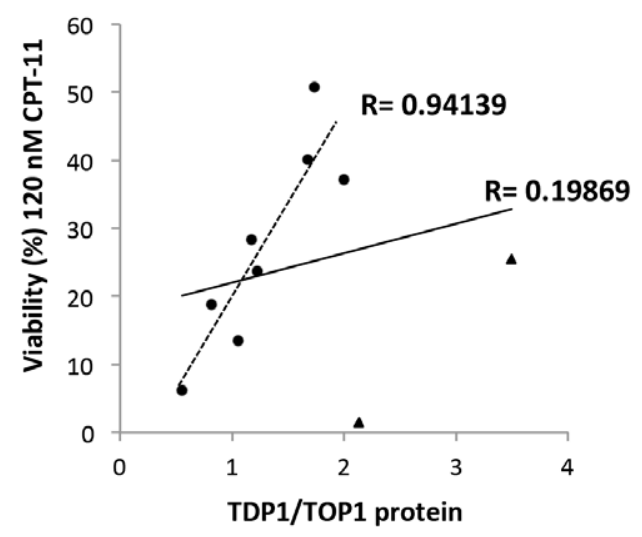

Figure 6: TDP1/TOP1 ratio predicts the cellular response of SCLC to topotecan. (A) Whole cell extracts $(40 \mu \mathrm{g})$ from the indicated SCLC cell lines were separated by $10 \%$ SDS-PAGE and immunblotted using antibodies against TDP1, TOP1 and actin. A quantitation of relative TDP1 (B) and TOP1 (C) protein level is shown. Data are the average of three independent experiments \pm STD normalised to HCC 33 for TDP1 and H510 for TOP1. (D) The indicated SCLC cell lines were seeded in triplicate at 10,000 cells per well in a 96-well plate in $100 \mu$ media containing the indicated concentration of topotecan (TPT) and incubated at $37{ }^{\circ} \mathrm{C}$ for 4 days. Viability was assessed by the CellTiter-Blue viability assay. Fluorescence was determined using a GloMax Multi Detection System (Promega, Southamption, UK) at excitation and emission wavelengths of $\lambda_{\mathrm{ex}} 560 \mathrm{~nm}$ and $\lambda_{\mathrm{em}} 590 \mathrm{~nm}$, respectively. For each cell line, percentage viability (\%) was calculated as [(Fluorescence read $\left.^{\text {treatment/Fluorescence read }}{ }^{\text {untreated }}\right)^{*} 100 \%$ ] and the graphical representation shows the results obtained from three independent experiments \pm STD. (E) Graphical representations depicting Pearson's correlation coefficient ( $r$ ) between SCLC viability at $120 \mathrm{nM}$ TPT treatment and TDP1/TOP1 levels. The solid line represents the correlation coefficient for all 10 SCLC cell lines whereas the dotted line represents the correlation coefficient for the eight SCLC cell lines HCC33, N417, H2171, H209, $\mathrm{H} 510, \mathrm{H} 82, \mathrm{H} 146$ and $\mathrm{H} 345$, excluding $\mathrm{H} 69$ and $\mathrm{H} 187$ (triangles), which exhibit high levels of TDP1 and an unexpected hypersensitivity to topotecan.

\section{Acknowledgement}

This work is funded by a Cancer Research UK grant to Sherif El-Khamisy and Simon Ward. El-Khamisy lab is additionally funded by a Wellcome Trust grant number WT091043 and a Fellowship from the Lister Institute of Preventative Medicine. The authors would like to thank members of the Sussex Translational Drug Discovery Group for helpful discussions. We thank Owen Wells for help with the DT40 cells and other members of the El-Khamisy lab for useful discussions.

\section{References}

1. Rosti G, Bevilacqua G, Bidoli P, Portalone L, Santo A, et al. (2006) Small cell lung cancer. Ann Oncol 17 Suppl 2: ii5-10.
2. van Meerbeeck JP, Fennell DA, De Ruysscher DK (2011) Small-cell lung cancer. Lancet 378: 1741-1755.

3. Jiang J, Liang X, Zhou X, Huang L, Huang R, et al. (2010) A meta-analysis of randomized controlled trials comparing irinotecan/platinum with etoposide/ platinum in patients with previously untreated extensive-stage small cell lung cancer. J Thorac Oncol 5: 867-873.

4. Lima JP, dos Santos LV, Sasse EC, Lima CS, Sasse AD (2010) Camptothecins compared with etoposide in combination with platinum analog in extensive stage small cell lung cancer: systematic review with meta-analysis. J Thorac Oncol 5: 1986-1993.

5. O’Brien ME, Ciuleanu TE, Tsekov H, Shparyk Y, Cuceviá B, et al. (2006) Phase 
Citation: Meisenberg C, Ward SE, Schmid P, El-Khamisy SF (2014) TDP1/TOP1 Ratio as a Promising Indicator for the Response of Small Cell Lung Cancer to Topotecan. J Cancer Sci Ther 6: 258-267. doi:10.4172/1948-5956.1000280

III trial comparing supportive care alone with supportive care with oral topotecan in patients with relapsed small-cell lung cancer. J Clin Oncol 24: 5441-5447.

6. Eckardt JR, von Pawel J, Pujol JL, Papai Z, Quoix E, et al. (2007) Phase III study of oral compared with intravenous topotecan as second-line therapy in small-cell lung cancer. J Clin Oncol 25: 2086-2092.

7. Kalemkerian GP, Akerley W, Bogner P, Borghaei H, Chow LQ, et al. (2013) Small cell lung cancer. J Natl Compr Canc Netw 11: 78-98.

8. Pommier Y, Pourquier P, Fan Y, Strumberg D (1998) Mechanism of action of eukaryotic DNA topoisomerase I and drugs targeted to the enzyme. Biochim Biophys Acta 1400: 83-105.

9. Wang X, Wang LK, Kingsbury WD, Johnson RK, Hecht SM (1998) Differential effects of camptothecin derivatives on topoisomerase I-mediated DNA structure modification. Biochemistry 37: 9399-9408.

10. Sordet O, Nakamura AJ, Redon CE, Pommier Y (2010) DNA double-strand breaks and ATM activation by transcription-blocking DNA lesions. Cell Cycle 9: 274-278.

11. Hudson JJR, Chiang S-C, Wells OS, Rookyard C, El-Khamisy SF (2012) SUMO modification of the neuroprotective protein TDP1 facilitates chromosomal single-strand break repair. Nature Communications 3: 733-813.

12. Alagoz M, Chiang SC, Sharma A, El-Khamisy SF (2013) ATM deficiency results in accumulation of DNA-topoisomerase I covalent intermediates in neural cells. PLoS One 8: e58239.

13. Gilbert DC, Chalmers AJ, El-Khamisy SF (2012) Topoisomerase I inhibition in colorectal cancer: biomarkers and therapeutic targets. Br J Cancer 106: 18-24.

14. Huang S-YN, Murai J, Dalla Rosa I, Dexheimer TS, Naumova A, et al. (2013) TDP1 repairs nuclear and mitochondrial DNA damage induced by chainterminating anticancer and antiviral nucleoside analogs. Nucleic Acids Res 41:7793-7803

15. Pommier Y, Huang SY, Gao R, Das BB, Murai J, et al. (2014) Tyrosyl-DNAphosphodiesterases (TDP1 and TDP2). DNA Repair (Amst) 19: 114-129.

16. Alagoz M, Wells OS, El-Khamisy SF (2014) TDP1 deficiency sensitizes human cells to base damage via distinct topoisomerase I and PARP mechanisms with potential applications for cancer therapy. Nucleic Acids Res 42: 3089-3103.

17. El-Khamisy SF, Saifi GM, Weinfeld M, Johansson F, Helleday T, et al. (2005) Defective DNA single-strand break repair in spinocerebellar ataxia with axonal neuropathy-1. Nature 434: 108-113.

18. Chiang SC, Carroll J, El-Khamisy SF (2010) TDP1 serine 81 promotes interaction with DNA ligase Illalpha and facilitates cell survival following DNA damage. Cell Cycle 9: 588-595

19. Das BB, Antony S, Gupta S, Dexheimer TS, Redon CE, et al. (2009) Optimal function of the DNA repair enzyme TDP1 requires its phosphorylation by ATM and/or DNA-PK. EMBO J 28: 3667-3680.

20. Interthal H, Chen HJ, Champoux JJ (2005) Human Tdp1 cleaves a broad spectrum of substrates, including phosphoamide linkages. J Biol Chem 280 : 36518-36528.

21. Walker S, Meisenberg C, Bibby RA, Askwith T, Williams G, et al. (2014) Development of an oligonucleotide-based fluorescence assay for the identification of tyrosyl-DNA phosphodiesterase 1 (TDP1) inhibitors. Anal Biochem 454: 17-22.

22. Huang SN, Pommier Y, Marchand C (2011) Tyrosyl-DNA Phosphodiesterase 1 (Tdp1) inhibitors. Expert Opin Ther Pat 21: 1285-1292.

23. Weidlich IE, Dexheimer T, Marchand C, Antony S, Pommier Y, et al. (2010)
Inhibitors of human tyrosyl-DNA phospodiesterase (hTdp1) developed by virtual screening using ligand-based pharmacophores. Bioorg Med Chem 18 : 182-189.

24. Marchand C, Lea WA, Jadhav A, Dexheimer TS, Austin CP, et al. (2009) Identification of phosphotyrosine mimetic inhibitors of human tyrosyl-DNA phosphodiesterase I by a novel AlphaScreen high-throughput assay. Mo Cancer Ther 8: 240-248.

25. Tomicic MT, Kaina B (2013) Topoisomerase degradation, DSB repair, p53 and IAPs in cancer cell resistance to camptothecin-like topoisomerase I inhibitors. Biochim Biophys Acta 1835: 11-27.

26. Nitiss JL, Wang JC (1996) Mechanisms of cell killing by drugs that trap covalent complexes between DNA topoisomerases and DNA. Mol Pharmacol 50: 10951102.

27. Zeng Z, Sharma A, Ju L, Murai J, Umans L, et al. (2012) TDP2 promotes repair of topoisomerase I-mediated DNA damage in the absence of TDP1. Nucleic Acids Res 40: 8371-8380.

28. El-Khamisy SF (2011) To live or to die: a matter of processing damaged DNA termini in neurons. EMBO Mol Med 3: 78-88.

29. Barthelmes HU, Habermeyer M, Christensen MO, Mielke C, Interthal $H$, et al. (2004) TDP1 overexpression in human cells counteracts DNA damage mediated by topoisomerases I and II. J Biol Chem 279: 55618-55625.

30. El-Khamisy SF, Katyal S, Patel P, Ju L, McKinnon PJ, et al. (2009) Synergistic decrease of DNA single-strand break repair rates in mouse neural cells lacking both Tdp1 and aprataxin. DNA Repair (Amst) 2009;8:760-806.

31. Das BB, Huang SY, Murai J, Rehman I, Amé JC, et al. (2014) PARP1-TDP1 coupling for the repair of topoisomerase l-induced DNA damage. Nucleic Acids Res 42: 4435-4449.

32. Gao R, Das BB, Chatterjee R, Abaan OD, Agama K, et al. (2014) Epigenetic and genetic inactivation of tyrosyl-DNA-phosphodiesterase 1 (TDP1) in human lung cancer cells from the NCl-60 panel. DNA Repair (Amst) 13: 1-9.

33. Interthal H, Chen HJ, Kehl-Fie TE, Zotzmann J, Leppard JB, et al. (2005) SCAN1 mutant Tdp1 accumulates the enzyme--DNA intermediate and causes camptothecin hypersensitivity. EMBO J 24: 2224-2233.

34. Miao ZH, Agama K, Sordet O, Povirk L, Kohn KW, et al. (2006) Hereditary ataxia SCAN1 cells are defective for the repair of transcription-dependent topoisomerase I cleavage complexes. DNA Repair (Amst) 5: 1489-1494.

35. Pommier Y, Barcelo JM, Rao VA, Sordet O, Jobson AG, et al. (2006) Repair of topoisomerase I-mediated DNA damage. Prog Nucleic Acid Res Mol Bio 81: 179-229.

36. Alagoz M, Gilbert DC, El-Khamisy S, Chalmers AJ (2012) DNA repair and resistance to topoisomerase I inhibitors: mechanisms, biomarkers and therapeutic targets. Curr Med Chem 19: 3874-3885.

37. Hamilton NK, Maizels N (2010) MRE11 function in response to topoisomerase poisons is independent of its function in double-strand break repair in Saccharomyces cerevisiae. PLoS One 5: e15387.

38. Deng C, Brown JA, You D, Brown JM (2005) Multiple endonucleases function to repair covalent topoisomerase I complexes in Saccharomyces cerevisiae. Genetics 170: 591-600.

39. Vance JR, Wilson TE (2002) Yeast Tdp1 and Rad1-Rad10 function as redundant pathways for repairing Top1 replicative damage. Proc Natl Acad Sci U S A 99: 13669-13674. 